\title{
Age-Related Changes in Serum/Plasma Biochemical Parameters of WHHLMI Rabbits
}

\author{
Satoshi YAMADA, Takashi ITO, Toshiaki TAMURA, and Masashi SHIOMI \\ Institute for Experimental Animals, Kobe University School of Medicine, \\ 7-5-1 Kusunoki-cho, Chuo-ku, Kobe 650-0017, Japan
}

\begin{abstract}
We developed myocardial infarction-prone rabbits (WHHLMI rabbits) by selectively breeding coronary atherosclerosis-prone WHHL rabbits. To examine the serum/ plasma biochemical parameters of this animal model, we assayed the lipid and glucose levels, and enzyme activities of WHHLMI rabbits from 2 to 26 months of age using solid phase analysis. The results showed a good correlation with those measured with a conventional method. The serum enzyme activities and lipid levels varied with aging despite almost no change in the plasma glucose levels. Gender differences were observed in the total cholesterol, triglyceride, and lactate dehydrogenase activity levels. The data on these serum/plasma biochemical parameters will be useful in studies of myocardial infarction or pharmacological studies using this model.
\end{abstract}

Key words: biochemical parameters, dry chemistry instrument, WHHLMI rabbits

We have carried out pre-clinical studies of several compounds using WHHL rabbits, which show spontaneous hypercholesterolemia, hyperinsulinemia and atherosclerosis $[4,12,15,16,21]$, to examine whether such compounds can reduce serum lipid levels, ameliorate insulin responses, or suppress atherosclerosis $[13-15,17-19,22]$. Although assays of serum/plasma biochemical parameters are important, there have been no reports on changes in these parameters with aging in this animal model or any other rabbit strain. Recently, we developed an animal model of spontaneous myocardial infarction (WHHLMI rabbit) by selectively breeding coronary atherosclerosis-prone WHHL rabbits [20]. This animal model suffers from myocardial infarction without any interventions and the findings of the coronary atheromatous plaques resemble those of patients with acute coronary syndromes [20]. Therefore, it should prove useful for pharmacological studies to prevent or suppress the development of myocardial infarction. Prior to using this animal model, however, it is important to assay basic serum/plasma biochemical parameters. Several serum/plasma biochemical parameters are considered to relate to the development of myocardial infarction. Therefore, an assay of serum/plasma biochemical parameters is also important in the evaluation of this animal model.

Assays with liquid reagents using a spectrophotometer, require a lot of time and repeated handling of the pipette. In addition, repeated handling of the pipette tends to cause large variance in the measurements. In

(Received 11 September 2003 / Accepted 17 December 2003)

Address corresponding: M. Shiomi, Institute for Experimental Animals, Kobe University School of Medicine, 7-5-1 Kusunoki-cho, Chuo-ku, Kobe 650-0017, Japan 
clinical tests, dry chemistry (solid phase analysis) instruments have been used for clinical analyses and the measurements taken with these instruments correlate well with those obtained with general liquid reagents using spectrophotometers or automatic clinical analyzers $[2,6,8]$. There are, however, few reports about the reliability of dry chemistry instruments for animal samples. Therefore, we examined the reliability of dry chemistry instruments for examining hyperlipidemic rabbit samples and assayed the serum/plasma biochemical parameters of WHHLMI rabbits at various ages. Regarding the mechanism of dry chemistry, concentration assays and enzyme activity measurements for serum/plasma biochemical parameters were carried out by measuring reflectance intensity at the appropriate wavelength for color-development in a dry and thin multiple layer-film analytical system containing all necessary reagents for colorimetric clinical analysis consisting of a spreading layer, a blocking layer, reagent layer(s), and a transparent layer [2, 6]. All aspects of animal experimentation and care were conducted according to the Guidelines of Animal Experimentation of Kobe University and the Guidelines for Animal Experimentation of the Japanese Association of Laboratory Animal Science.

To examine whether dry chemistry is applicable to the assay of serum/plasma biochemical parameters of hyperlipidemic WHHLMI rabbits, we carried out a correlation analysis between measurements made with dry chemistry methods using a Fuji Dri-Chem 3500SV (Fuji Photo Film Co., Ltd., Tokyo, Japan) and those determined with liquid reagents using a Hitachi Automatic Clinical Analyzer 7170 (Hitachi Ltd., Tokyo, Japan). According to the instructions for each multiple layerfilm, we assayed the concentration of serum total cholesterol, triglyceride, and plasma glucose, and serum enzyme activities of alkaline phosphatase (ALP), creatine phosphokinase (CPK), aspartate aminotransferase/glutamic-oxaloacetic transaminase (AST/GOT), alanine aminotransferase/glutamic-pyruvic transaminase (ALT/GPT), and lactate dehydrogenase (LDH) of male and female WHHLMI rabbits at the ages of 2 months, 6 months, 10-12 months, 15-18 months, and 21-26 months. In the correlation analysis of data for dry chemistry and liquid reagent methods, we prepared serum/plasma samples of various concentrations/enzyme activities by diluting samples showing high concentra- tions/enzyme activities with samples showing low concentrations/enzyme activities at various ratios. In the assay of plasma glucose levels, rabbits were administered saline containing sucrose at a $10 \%$ concentration into the marginal ear vein and the plasma samples were prepared using blood samples obtained $10 \mathrm{~min}$ after sucrose administration according to a method reported previously [17]. As shown in Fig. 1, measurements of the serum/plasma biochemical parameters made with the dry chemistry method showed a good correlation with those obtained with liquid reagents. These results clearly demonstrate that measurements made with the dry chemistry instrument are reliable for sera from hyperlipidemic WHHLMI rabbits. In assays with an automatic dry chemistry instrument, operators do not need to handle a pipette, except for sample dilution. Therefore, compared to assays using liquid phase reagents with a spectrophotometer, using an automatic dry chemistry instrument will reduce the work load in assays of serum/plasma biochemical parameters with a large number of samples in screening or pharmacological studies, and will reduce the variance of measurements. In addition, one can shorten the assay time and reduce sample volume by using a solid phase assay system, compared to a liquid phase assay.

Using the dry chemistry method, we examined serum/plasma biochemical parameters of male and female WHHLMI rabbits at various ages (Fig. 2). Levels of total cholesterol (Panel A) decreased with aging and male cholesterol levels were significantly lower than female levels beyond 6 months of age. An age-related reduction in serum triglyceride levels was observed in male rabbits and the difference between the genders was significant (Panel B). In humans, serum/plasma lipid levels increase with aging and are lower in females before menopause. Although there is no evidence to explain this difference in changes of serum lipid levels between humans and WHHLMI rabbits, the difference in dietary habits between these species may relate to changes in the serum lipid levels. Although serum cholesterol levels of newborn rabbits $(80-100$ $\mathrm{mg} / \mathrm{dl})[3,10]$ are similar to those of newborn infants $(50-70 \mathrm{mg} / \mathrm{dl})$ [1,23], humans ingest animal fats, which relate to an increase in serum lipid levels. The gender difference in serum lipid levels of humans is considered to be due to the estrogen function in females. In rabbits, however, the estrus cycle is unclear and the 


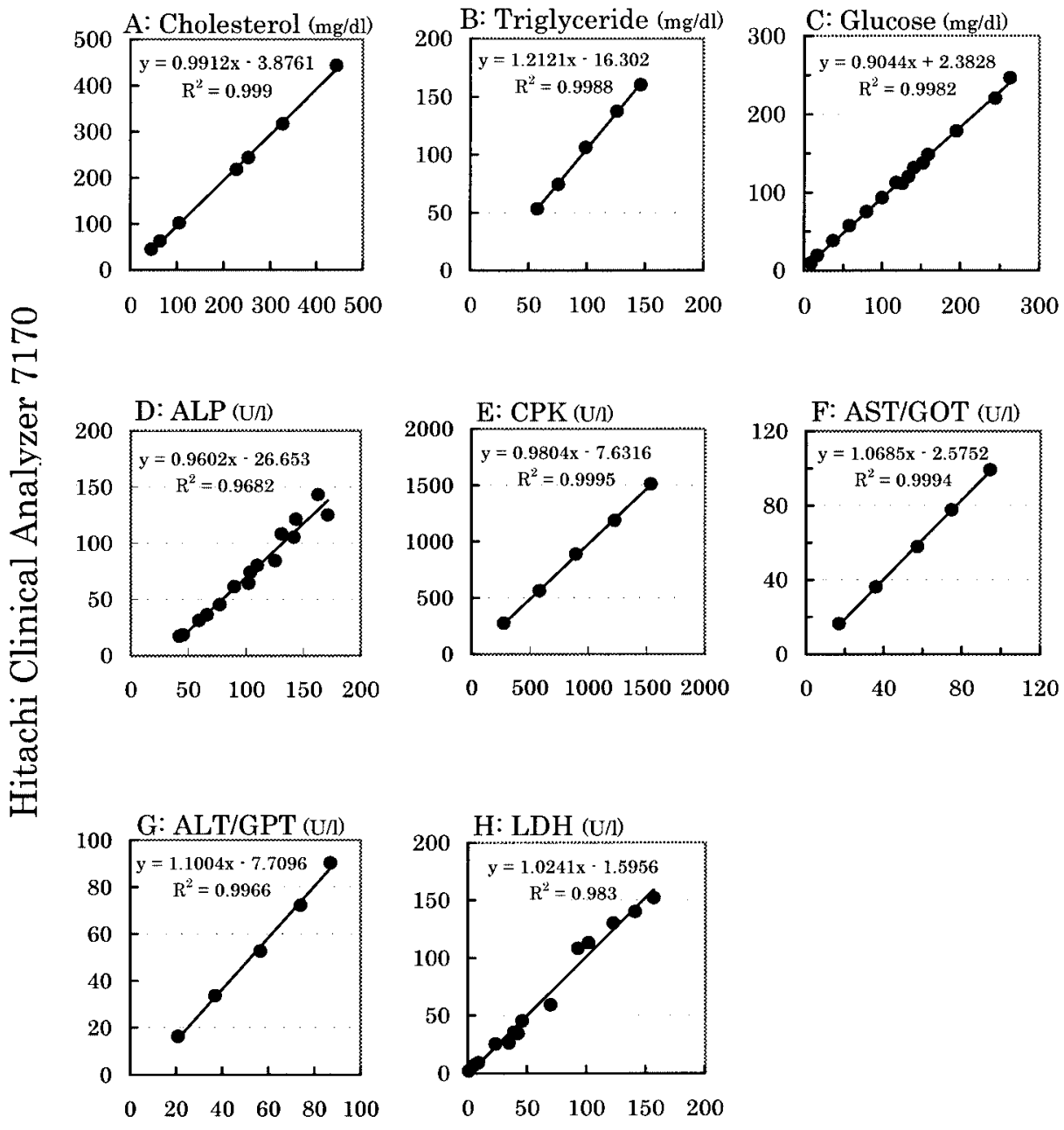

\section{Fuji Dri-Chem 3500SV}

Fig. 1. Correlation of measurements of serum/plasma biochemical parameters made with solid phase analysis using an automatic dry chemistry instrument (Fuji Dri-Chem 3500SV) to those made with liquid phase analysis using standard liquid reagent methods with an automatic Clinical Analyzer (Hitachi Automatic Clinical Analyzer 7170). With the liquid reagent approach, serum lipid levels and plasma glucose levels were assayed with enzymatic methods and serum enzyme activities were assayed with the standard methods of the Japanese Society of Clinical Chemistry or the standard methods of the International Federation of Clinical Chemistry. A, Total cholesterol ( $n=7)$; B, Triglyceride ( $n=9)$; C, Glucose ( $n=15)$; $\mathrm{D}$, alkaline phosphatase (ALP, $\mathrm{n}=24)$ ); $\mathrm{E}$, creatine phosphokinase $(\mathrm{CPK}, \mathrm{n}=5)$ ); $\mathrm{F}$, aspartate aminotransferase/glutamic-oxaloacetic transaminase (AST/GOT, $n=5$ ): $\mathrm{G}$, alanine aminotransferase/glutamic-pyruvic transaminase (ALT/GPT, n=5); H, lactate dehydrogenase (LDH, $\mathrm{n}=24)$. The correlation coefficients were calculated with Pearson's method.

serum $17 \beta$-estradiol levels of WHHL rabbits did not show significant fluctuation and the intramuscular injection of various estrogens did not reduce serum cholesterol levels of WHHL rabbits despite the hypocholesterolemic effects of estrogens in humans
[11]. This result suggests that the effects of serum $17 \beta$-estradiol on serum cholesterol levels may be weak in WHHLMI rabbits. The plasma glucose levels were almost constant below 24 months of age and there were no gender differences except at 2 months of age (Panel 

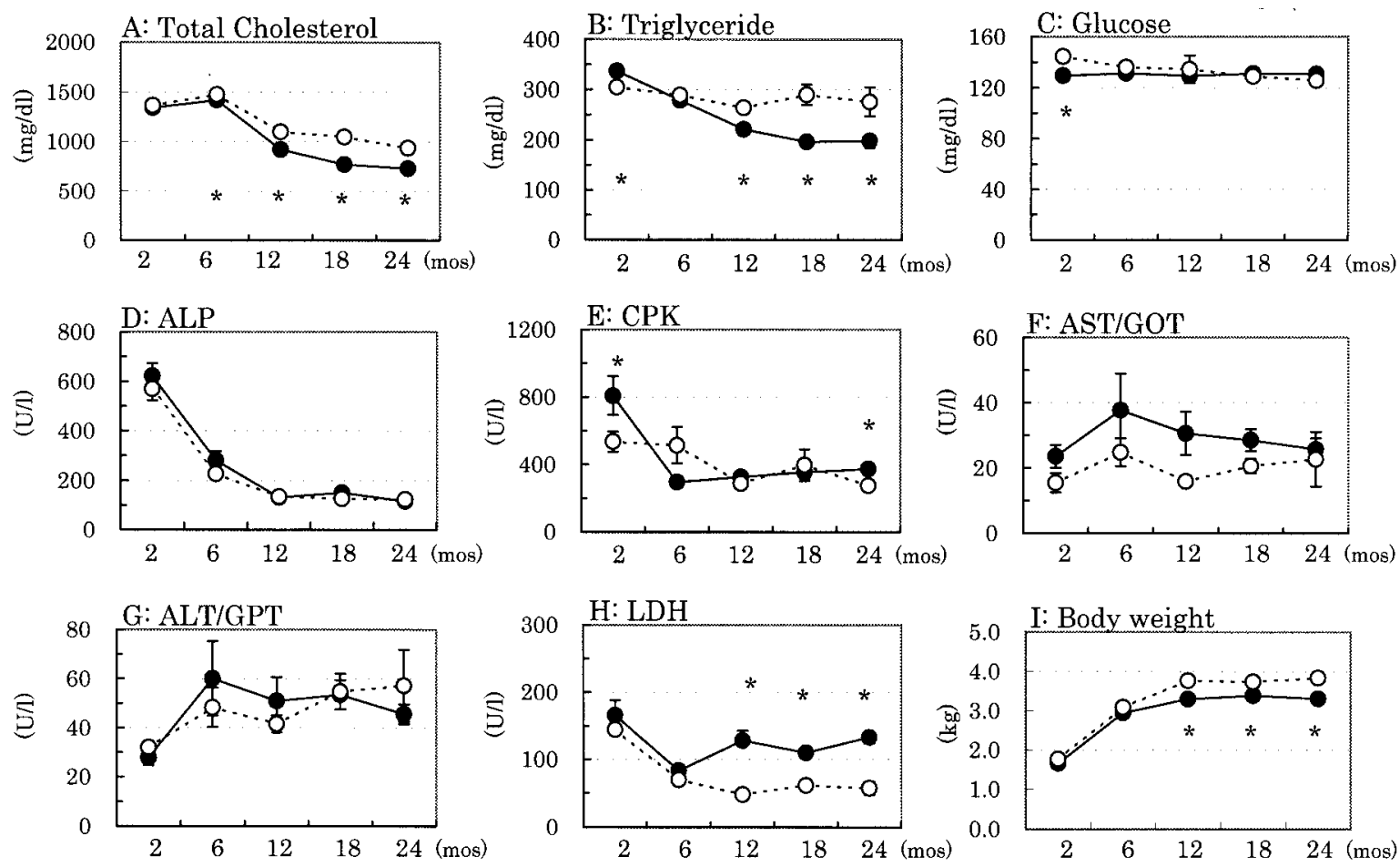

Fig. 2. Serum/plasma biochemical parameters of male $(\mathbf{O})$ and female $(\bigcirc)$ WHHLMI rabbits assayed with a dry chemistry instrument (Fuji Dri-Chem 3500SV) and body weight. Numbers of animals examined were 134 at 2 months, 98 at 6 months, 59 at 12 months, 42 at 18 months, and 25 at 24 months for serum lipid levels and 10 animals each for the other parameters. A, Total cholesterol; B, Triglyceride; C, Plasma Glucose; D, alkaline phosphatase (ALP); E, creatine phosphokinase (CPK); F, aspartate aminotransferase/glutamic-oxaloacetic transaminase (AST/GOT): G, alanine aminotransferase/glutamic-pyruvic transaminase (ALT/GPT); H, lactate dehydrogenase (LDH); I, body weight. Data are represented as the mean \pm the standard error of the mean. Statistical analyses were carried out with Student's t-test or Aspin-Welch's t-test and statistical significance was set at $\mathrm{P}<0.05$. *, $\mathrm{P}<0.05$.

C). ALP activities decreased from 2 to 12 months of age and were then constant (Panel D). This finding is similar to that in humans $[7,24]$. Serum CPK activity levels were very high and varied markedly at each age (Panel E). In a preliminary analysis of the isozymes with electrophoresis, the MM-type fraction, which is derived from striated muscle, showed a large peak but the peak of MB-type, which is derived from myocardium, is very small (unpublished results of Shiomi, M.). The relatively high serum CPK levels in 2-month-old rabbits may be due to the activity of young rabbits because serum CPK levels are increased easily by physical exercise [7, 24]. This suggests that very high serum CPK levels do not always reflect the development of myocardial infarction and that it is necessary to be careful in evaluating serum CPK levels in rabbits. For AST/GOT and ALT/GPT, activity levels increased from
2 to 6 months of age and then remained relatively constant (Panel F and G). The levels of LDH activity were high at 2 months of age compared to 6 months or older and were significantly higher in males than females from age 12 months (Panel $\mathrm{H}$ ). These findings are similar to those in humans [7, 24]. In suckling rabbits, the serum cholesterol levels were high compared to adult rabbits $[3,10]$ and decreased slightly at 2 months of age [3]. This suggests that the influence of suckling remains at 2 months of age, and the high levels of ALP and LDH and low levels of AST/GOT and ALT/GPT at 2 months of age may also be due to the influence of suckling. In addition, the increase in body weight was significantly large in females (Panel I). Similar findings were observed in white rabbits [5] and Himalayan rabbits [9], however, there is no explanation for this gender difference. 
In the present study, we assayed serum/plasma biochemical parameters in WHHLMI rabbits, which are important in pharmacological studies and studies of myocardial infarction. The data are the first of their type for WHHLMI rabbits. Although several parameters, such as CPK, LDH, and AST/GOT, are used as markers of myocardial infarction in clinical examinations, increases in these parameters are observed at only the acute phase and serum levels are also increased by physiological exercise $[7,24]$, therefore, it is necessary to be careful when interpreting abnormal data. However, the present results may be useful for the analysis of sudden coronary ischemic decease if the sera are obtained immediately after the coronary event. Therefore, information about changes in serum/plasma biochemical parameters with aging and gender differences will probably be useful in studies of myocardial infarction and preclinical pharmacological examinations.

\section{Acknowledgments}

This work was supported in part by research grants from Takeda Chemical Industries Ltd. We acknowledge Fuji Photo Film Co., Ltd. for their technical assistance.

\section{References}

1. Bersohn, I. and Wayburne, S. 1956. Am. J. Clin. Nutr. 4: 117-123.

2. Curme, H.G., Columbus, R.L., Dappen, G.M., Eder, T.W., Fellows, W.D., Figueras, J., Glover, C.P., Goffe, C.A., Hill, D.E., Lawton, W.H., Muka, E.J., Pinney, J.-E., Rand, R.N., Sanford, K.J., and Wu, T.W. 1978. Clin. Chem. 24: 13351342.

3. Dietschy, J.M., Kita, T., Suckling, K.E., Goldstein, J.L., and Brown, M.S. 1983. J. Lipid. Res. 24: 469-480.

4. Goldstein, J.L., Kita, T., and Brown, M.S. 1983. N. Engl.
J. Med. 309: 288-296.

5. Hosaka, K. 1956. Bull. Exp. Anim. 5: 32-35 (in Japanese).

6. Kasahara, K. 1997. Jpn. J. Clin. Pathol. 106: 12-22 (in Japanese)

7. Kato, H., Mitsudome, A., Hara, T., and Yoshida, I. Eds. 2001. "Beddo Saido No Shoni No Mikata" 54-57, Nanzando, Tokyo, Japan (in Japanese).

8. Ohkubo, A., Kamei, S., Yamanaka, M., Arai, F., Kitajima, M., and Kondo, A. 1981. Clin. Chem. 27: 1287-1290.

9. Matsuo, A., Kazuta, H., and Kast, A. 1981. Exp. Anim. 30: 269-273.

10. Popjak, G. 1946. J. Physiol. 105; 236-254.

11. Shiomi, M. 1987. (Dissertation, Otsu-261), Azabu University School of Veterinary Medicine (in Japanese).

12. Shiomi, M. 2003. WHHL rabbit Web site: http:// www.med.kobe-u.ac.jp/iea/whhl-1.html.

13. Shiomi, M. and Ito, T. 1994. Metabolism 43: 559-564.

14. Shiomi, M., Ito, T., Fujioka, T., and Tsujita, Y. 2000. Metabolism 49: 552-556.

15. Shiomi, M., Ito, T., Hirouchi, Y., and Enomoto, M. 2001. Atherosclerosis 157: 75-84.

16. Shiomi, M., Ito, T., Shiraishi, M., and Watanabe, Y. 1992. Atherosclerosis 96: 43-52.

17. Shiomi, M., Ito, T., Tsukada, T., Tsujita, Y., and Horikoshi, H. 1999. Atherosclerosis 142: 345-353.

18. Shiomi, M., Ito, T., Tsukada, T., Yata, T., Watanabe, Y., Tsujita, Y., Fukami, M., Fukushige, J., Hosokawa, T., and Tamura, A. 1995. Arterioscler Thromb. Vasc. Biol. 15: 1938-1944.

19. Shiomi, M., Ito, T., Watanabe, Y., Tsujita, Y., Kuroda, M., Arai, M., Fukami, M., Fukushige, J., and Tamura, A. 1990. Atherosclerosis 83: 69-80.

20. Shiomi, M., Ito, T., Yamada, S., Kawashima, S., and Fan, J. 2003. Arterioscler Thromb. Vasc. Biol. 23: 1239-1244.

21. Watanabe, Y. 1980. Atherosclerosis 36: 261-268.

22. Watanabe, Y., Ito, T., Shiomi, M., Tsujita, Y., Kuroda, M., Arai, M., Fukami, M., and Tamura, A. 1988. Biochim. Biophys. Acta. 960: 294-302.

23. Webber, L.S., Frank, G.C., Smoak, C.G., Freedman, D.S., and Berenson, G.S. 1987. Pediatrics 80: 767-787.

24. Zaidan Houjin Nippon Koushu Eisei Kyokai. 1997. "Nipponjin Shouni No Rinsho Kensa Kijunchi" 15-67. Zaidan Houjin Nippon Koshu Eisei Kyokai, Tokyo, Japan (in Japanese). 